\title{
Aportes de lectores y lectoras de tesis doctoral como mediación pedagógica en la formación de personas investigadoras
}

Torres-Frías, José de la Cruz; Moreno-Bayardo, María Guadalupe; Jiménez-Mora, José Margarito Aportes de lectores y lectoras de tesis doctoral como mediación pedagógica en la formación de personas investigadoras

Revista Educación, vol. 42, núm. 1, 2018

Universidad de Costa Rica, Costa Rica

Disponible en: http://www.redalyc.org/articulo.oa?id=44051918014

DOI: https://dx.doi.org/10.15517/revedu.v42i1.22970

Esta obra está bajo una Licencia Creative Commons Atribución-NoComercial-SinDerivar 3.0 Internacional. 


\section{Aportes de lectores y lectoras de tesis doctoral como mediación pedagógica en la formación de personas investigadoras}

\section{The Contributions of Doctoral Thesis Readers as Pedagogical Mediation in the Training of Researchers}

José de la Cruz Torres-Frías [1]

Universidad de Guadalajara, México

cruzfrias@gmail.com

Maria Guadalupe Moreno-Bayardo [2]

Universidad de Guadalajara, México

gpemor98@hotmail.com

José Margarito Jiménez-Mora [3]

Universidad de Guadalajara, México

pepe_dw@hotmail.com
DOI: https://dx.doi.org/10.15517/revedu.v42i1.22970

Redalyc: http://www.redalyc.org/articulo.oa?id=44051918014

\section{Resumen:}

Este documento se desprende de una investigación más amplia [4]. Su propósito es dar cuenta de la naturaleza del contenido de las intervenciones del personal lector académico y el papel que juega en la formación de personas investigadoras en doctorados en educación. Se trata de un estudio de caso múltiple que recupera 100 reportes escritos de 9 lectores y lectoras que laboran en tres doctorados en educación en el Centro-Occidente de México; la totalidad con grado de doctorado. Los reportes se analizaron con apoyo en principios del análisis de contenido. Los hallazgos dan cuenta de algunos rasgos del personal investigador, ciertas pistas del oficio como la de aportar conocimiento nuevo, explicitar el involucramiento personal con el objeto de estudio, atender la escritura académica, problematizar, articular, desplegar la vigilancia epistemológica, hacer afirmaciones con sustento, entre otras. Estos rasgos identificados, en cierto grado son reflejo del deber ser de la investigación que han internalizado los lectores y lectoras, quienes distan mucho de ser considerados como la voz de una normativa o manual de investigación. El papel lector va más allá, se trata de una verdadera mediación pedagógica para formar en investigación, no siempre valorada en su justa dimensión, porque existe personal académico que la realiza a la ligera, la usa para exhibir al estudiantado o criticar a los pares por motivos como el juego de posiciones de poder en un campo científico.

PALABRAS ClaVE: educación, posgrado, doctorado, lectores, formación de personal Investigador.

\section{Abstract:}

\section{NotAS DE AUTOR}

[1] Cuenta con estudios de Doctorado en Educación y posdoctorado en Enseñanza de las Ciencias, adscrito al Centro Universitario del Norte de la Universidad de Guadalajara, México. Es miembro del Sistema Nacional de Investigadores y cuenta con el reconocimiento al perfil deseable del PRODEP. Su línea de investigación aborda objetos de estudio relacionados con la formación para la investigación en Educación Superior y posgrado. Su publicación más reciente es: El papel de la tutoría en la formación de habitus científicos en estudiantes de doctorado en educación.

[2] Obtuvo el grado de Doctorado en Educación por la Universidad Autónoma de Guadalajara. Es profesora investigadora titular C de tiempo completo en la Universidad de Guadalajara, México, en donde ha desempeñado las funciones de Coordinadora del Doctorado en Educación y jefa del Departamento de Estudios en Educación. Fue coordinadora de Formación y Actualización de Docentes en la Secretaría de Educación Jalisco, directora general de Posgrado e Investigación Educativa en la misma Secretaría, así como colaboradora de diversos equipos de trabajo en la Secretaría de Educación Pública. Es miembro del Sistema Nacional de Investigadores en el Nivel II, y del Consejo Mexicano de Investigación Educativa. Sus líneas de investigación son la formación para la investigación, la formación de docentes y la enseñanza de las matemáticas. Entre sus obras publicadas, se encuentran más de 100 artículos en revistas educativas, así como 20 libros y 18 capítulos de libro, la mayoría de ellos relacionados con la formación para la investigación y los posgrados en educación. Algunos de sus libros publicados son: Formación para la investigación centrada en el desarrollo de habilidades; Historias de formación para la investigación en doctorados en educación; El discurso de los lectores académicos como mediación para formar investigadores en el posgrado

[3] Maestro en Investigación en Ciencias de la Educación por la Universidad de Guadalajara. Investigador del Departamento de Estudios en Educación de la UdeG. Publicación reciente: "El discurso de los lectores académicos como mediación en la formación para la investigación en el posgrado", UdeG; "Aportaciones de los lectores académicos para el aprendizaje de la investigación educativa", Revista Electrónica Educare, Universidad de Costa Rica, abril-enero 2016 
The content of this document is derived from a broader research investigation [5]. Its purpose is to give an account of the nature of the content of the interventions of academic readers and the role they play in the training for the profession of researcher in doctorates in education. It is a multiple case study that retrieved 100 written reports from 9 readers, who work in three doctoral programs in education in the Center-West of Mexico, all of them with doctorate degrees. The analysis of the reports was done with a basis in the principles of content analysis. The findings reveal some traits of the researcher, certain clues to the profession such as providing new knowledge, explaining personal involvement with the object of study, attending to academic writing, problematizing, articulating, deploying epistemological surveillance, making affirmations with sustenance, among others. To a certain degree these identified traits are a reflection of the requirements of doing research. They are considered far from being merely the voice of a formal guideline or a research manual. The role of the reader goes beyond that, it is a true pedagogical mediation for the training of researchers, not always valued to the proper degree because there are academics who do it lightly, using it to show off to students, or to criticize peers for motives such as the playing of power games in a specific scientific field.

KEYWORDS: education, postgraduate, PHD, readers, research training.

\section{INTRODUCCIÓN}

En el contexto académico en general, y en el ámbito del posgrado en particular, circulan ideas compartidas entre el personal de investigación sobre la formación para la investigación, que en gran medida, orientan los procesos, las prácticas y las estrategias de formación que se implementan en ese contexto educativo: a) que la investigación es un oficio, en tanto que la generación de conocimiento supone una artesanía intelectual; b) que el oficio de investigador se aprende investigando; c) que ese aprender haciendo no ocurre en solitario, sino con el apoyo y la mediación de investigadores e investigadoras en activo con producción científica; d) que en la interacción entre docente y aprendiz se realiza una formación con carácter artesanal; e) que en dicha formación se promueve el desarrollo e interiorización de ciertas disposiciones académicas que dan forma relativa al habitus científico y, f) que es en el nivel de estudio de doctorado donde se lleva a cabo la formación escolarizada más sistemática e intencionada para el oficio de investigación.

Para quienes fungimos como autoría de este texto, los estudios de doctorado, al estar orientados a la formación de personal investigador (como lo es en el caso de México, por lo menos hasta 2014), abarcan un proyecto de vida profesional y personal, que trasciende las fronteras de "lo académico" y de un cuerpo de conocimiento. Va más allá de un proyecto científico que despunta en una contribución original al conocimiento (Trafford y Leshmen, 2009). Comprende, pues, la interiorización de un modo de vida profesional, un modo de ser y hacer, una manera particular de posicionarse frente a la vida, la cultura, la sociedad y el mundo en general, y transformarlo de manera progresiva en un estilo de vida personal.

El planteamiento anterior sugiere que el nivel de estudio de doctorado, sea percibido como campo abierto de posibilidades variadas de formación, aprendizaje y crecimiento intelectual, académico, personal y experiencial; donde el investigador o la investigadora en formación tiene la oportunidad de realizar un proyecto científico en un campo de conocimiento, con un alto grado de autonomía e independencia intelectual, en un nivel científico aceptable para los sujetos miembros de la comunidad académica erudita (Dietz, Jansen y Wadee, 2006), además de ser capaz de integrarse de manera activa a ella. En dicho espacio académico participan diversos grupos investigadores como formadores, en algunos casos, responsables de un seminario o taller, como profesoras o profesores invitados, como personal de tutoría de alguien que se está doctorando, o como personal lector de tesis.

Aunque aquí no se niega el papel protagónico de la tutoría o asesoramiento de tesis en la formación para el oficio de personal investigador en doctorado, ni las contribuciones que realizan sus docentes responsables de algún seminario del plan de estudios del doctorado que se cursa; la mirada está puesta en la función formadora, y en las contribuciones que pueden realizar quienes participan como lectores o lectoras de tesis en doctorado (a quienes se nombra, también, como personal investigador-lector o lector académico) en tanto agentes de la investigación e integrantes de algún comité tutorial, responsables de contribuir a la formación 
del estudiantado vía retroalimentación critica de los avances de investigación reportados por los sujetos doctorandos en los coloquios de fin de semestre.

Por lo menos en México, los programas de doctorado en educación orientados a formar personal investigador -en especial en los doctorados seleccionados para este estudio-, suele existir una especie de relación entre el semestre que cursa el estudiantado y el producto que se espera realice y presente en los espacios académicos denominados coloquios. Así, por ejemplo, hay un semestre donde el producto esperado por el personal formador es el estado del conocimiento, otro donde se espera la construcción del objeto de estudio, y así sucesivamente hasta que presente el proyecto de tesis doctoral. En ese marco, resulta "natural", que cada semestre, los comentarios del personal lector académico se orienten, sobre todo, al aspecto que constituye el foco del avance de investigación en turno; aunque se reconoce que cada quien conoce lo que el estudiantado ha producido en semestres previos al formar parte de su comité tutorial a lo largo de todo el doctorado.

Como señalan Holbrook, Bourke, Lovat y Fairbairn (2008), quienes fungen como personal tutor y lector académico juegan un papel crucial en la definición y formación de las prácticas en sus disciplinas, incluso, inciden en el qué y cómo los candidatos y las candidatas requieren aprender, en el marco de un programa de doctorado, para alcanzar el éxito académico, de ahí su carácter de agente de la investigación. En ese sentido, el personal lector académico no solamente cumple la función de evaluador o sancionador del producto escrito que reciben para su revisión, sino que además tienen una función de enseñanza que posibilita que se les considere como sujetos formadores de personal investigador en el posgrado.

Desde esta perspectiva, el presente documento expone los hallazgos de una investigación focalizada en el discurso de personal lector académico como mediación para formar investigadores e investigadoras en el posgrado. El propósito del estudio fue dar cuenta de la naturaleza del contenido de sus intervenciones y el papel que juegan en la formación de personas investigadoras en doctorados en educación. El documento está constituido por cinco secciones: 1) Los lectores y lectoras de tesis de doctorado en la formación de personal de investigación, 2) los lectores y lectoras de tesis como agentes de la investigación en un campo científico, 3) procedimientos metodológicos, 4) análisis y discusión de resultados, 5) a manera de cierre. A continuación, se desarrolla cada uno de ellos.

\section{1.- LOS LECTORES Y LECTORAS DE TESIS DE DOCTORADO EN LA FORMACIÓN DE PERSONAL DE INVESTIGACIÓN}

Durante la formación para la investigación, en el marco de los estudios de posgrado, en especial en el doctorado, la figura académica de director o directora de tesis, también conocida como tutor o tutora (en otros países supervisor o supervisora), asume un papel protagónico en la formación del sujeto doctorando a su cargo. Su función principal consiste en acompañarlo de manera directa e intensiva durante todo ese proceso; conocer y evaluar en cada etapa su avance de investigación; asistirlo en la búsqueda de experiencias que apoyen y complementen su formación (seminarios, talleres, congresos, estancias académicas); dialogar con él sobre sus dudas y dificultades, así como asesorarlo en la toma de decisiones. En sí, se trata de atender de manera personalizada sus necesidades de formación a lo largo de su permanencia en el doctorado, y ser el principal aval para el otorgamiento del grado.

Sin embargo, hay que reconocer que quien participa como lector o lectora de tesis o personal lector académico (conocido en otros países como examinador o jurado) en ese contexto educativo, juega un papel relevante por la contribución académica que, desde su experiencia en investigación puede hacer a la formación del estudiantado. El personal lector de tesis puede o no estar adscrito al programa académico de formación, ser institucionalmente interno o externo, nacional o internacional. No obstante, como sugiere Jack (2002), su participación habrá de sustentarse en sus aportes de conocimiento, en el dominio de los estándares de calidad solicitados en ese campo, en el conocimiento que tiene sobre el campo temático en el que se inscribe el objeto 
de estudio, y el dominio experto de las aproximaciones teórico-metodológicas validadas como pertinentes en la comunidad científica de referencia.

A esta figura académica (lector o lectora de tesis), se le atribuye la función de colaborar con el personal tutor en la trayectoria de formación del sujeto doctorando, por eso, es parte del comité tutorial conformado para la estudiante o el estudiante asignado (Moreno, Torres y Jiménez 2013). En ese sentido, puede asistir al personal tutor y al tutorado o tutorada en cualquiera de las actividades de formación, al proporcionar experiencias de desarrollo, pistas y retroalimentaciones; sin embargo, una de sus responsabilidades principales es dar lectura, evaluar, hacer observaciones y sugerencias a los avances de investigación -y a la versión final de la tesis- que cada estudiante presenta en el coloquio de fin de semestre. La extensión, tipo y tono de los comentarios presentados en sus reportes escritos proporciona evidencia concreta del compromiso, la profundidad y la calidad de la revisión que ha desplegado, al valorar la calidad del producto entregado (Bourke, Hattie y Anderson, citado por Holbrook, et al., 2008).

Como argumentan Mullins y Killey (2002), el personal lector asume el documento presentado para su revisión como un todo. En él se valora la calidad con que fue desarrollado cada uno de los momentos de la investigación, así como su respectiva articulación, sin degradar su calidad, rigor y lógica argumentativa. Esto es, buscan percibir en la producción académica de cada estudiante que ha logrado desarrollar "una tesis doctoral coherente y metodológicamente plausible" (Trafford y Leshem, 2009, p. 305) que ha transitado de un estado novato o de aprendiz del oficio de investigación al de agente legítimo, al de persona experta en la generación de conocimiento por vía de la investigación.

Con base en esta serie de acciones, se considera que el personal lector de tesis, asignado a cada estudiante, aporta una "mirada calificada" a sus avances de investigación, y participa en la decisión de si el producto que presentan cumple los requisitos institucionales, y tiene las características y el nivel de calidad requerido para ser presentada como tesis, y sustentar la obtención del grado académico correspondiente. En ese sentido, juega el papel de "árbitro" o "portero" de un campo científico, dado que definen, imponen y exigen los estándares de calidad que requieren estar presentes en las producciones académicas de los doctorados. Por ello, sus señalamientos y orientaciones, en el marco de los coloquios, pueden considerarse como "pistas" valiosas sobre el oficio de investigador o investigadora.

\section{2.- LOS LECTORES Y LECTORAS DE TESIS COMO AGENTES DE LA INVESTIGACIÓN EN UN CAMPO CIENTÍFICO}

En páginas anteriores se ha aludido a términos como campo científico, habitus científico y agentes, que provienen de la teoría de los campos de producción simbólica construida por Pierre Bourdieu a lo largo de su obra, la cual se retoma aquí como perspectiva de base que permite comprender y explicar los datos empíricos que respaldan este trabajo.

La idea de campo científico remite a un conjunto de disciplinas de conocimiento, concebidas como campos locales que comparten unos intereses y unos principios mínimos (Bourdieu, 2003). Caracterizadas por su relativa autonomía "reflejada" en su capacidad de retraducir las presiones externas (políticas, económicas, administrativas) en beneficios internos (estímulos económicos, adquisición de equipo tecnológico, prestigio o mejores condiciones laborales), y en su relativo grado de libertad para definir los requisitos de ingreso al campo y "elegir" los habitus adecuados para realizar sus propias prácticas, la de investigación (Bourdieu, 1976). En su interior existe un conjunto de personal investigador e instituciones científicas que fungen como agentes de la investigación y de la formación, circula un capital cultural eficiente concebido como capital científico, predomina un grado más o menos elevado de concentración de ese capital, y en él se exigen de manera tácita unos habitus científicos.

También se aprecia que, como campo científico, en ellas perviven dos formas de poder asociadas a dos especies de capital científico: el capital administrativo y el capital científico "puro", los cuales se sostienen por 
mecanismos de acumulación y transmisión diferentes y son susceptibles de ser acumuladas por un mismo agente (personal investigador). El primero (personal administrativo) está ligado a la ocupación de posiciones de prestigio en instituciones científicas, comisiones y comités. Se adquiere vía estrategias políticas y se puede transferir vía reglas de orden burocrático como el concurso de "elección". Genera un poder institucional temporal y un poder sobre los medios de producción y reproducción que contribuye a afianzar dicha posición. En cambio, el capital científico puro tiene como base principal el reconocimiento individual -objetivado e institucionalizado- de los pares investigadores (competidores) por las contribuciones de conocimiento para el avance de la ciencia. Su acumulación se logra por la publicación de aportes de conocimiento, invenciones o descubrimientos (Bourdieu, 2003).

El capital científico puro, señala Bourdieu (2003), abarca cierto colectivo de conceptos especializados y una tradición científica de presupuestos compartidos; acuerdos sobre la aceptación de la prueba/evidencia empírica como "arbitraje final"; formas racionalizadas, formalizadas y estandarizadas de pensamiento; maneras de conocer un objeto producto de un arsenal de teorías complejas, métodos, técnicas e instrumentos de recolección y análisis de datos, empleados de manera articulada con la coherencia de las disciplinas, las censuras del campo y la orquestación invisible de los habitus científicos en el oficio investigador, cuyo dominio constituye la frontera de la disciplina y el derecho de admisión al campo (Bourdieu, 2003). Por ello, se afirma que cada disciplina tiene su manera de problematizar, de construir observables, teorías y marcos de fundamentación conceptual, de comprobar hipótesis o supuestos. Hay especificidad en el conocimiento, la tradición, la costumbre y la práctica enseñada, en las creencias, principios morales y normas de conducta (ethos), y en las formas lingüísticas y simbólicas de comunicación.

Del señalamiento anterior, se desprende que el campo científico, además de ser un lugar para la generación de conocimiento por vía de la investigación, también es un campo de lucha entre personal investigador, donde se enfrentan construcciones sociales competidoras, argumentos que buscan el reconocimiento de esa manera de conocer, la autoría de ideas (aportes de conocimiento), la notoriedad científica y la conquista del monopolio de esa autoridad científica que "concede" el "derecho" de hablar con autoridad sobre el quehacer científico.

Respecto al habitus cientifico, Bourdieu (1976, p.96) lo refiere como "sistema de esquemas generadores de percepción, de apreciación y de acción que son el producto de una forma específica de acción pedagógica y que vuelven posible la elección de los objetos, la solución de los problemas y la evaluación de las soluciones". También lo concibe como "principios de producción de prácticas", "sistema de disposiciones generadoras transportables", "teoría realizada e incorporada" y "sentido práctico de los problemas que se van a tratar y unas maneras adecuadas de ser tratados" (Bourdieu, 2003, pp. 73-79).

Se trata, pues, de concepciones científicas condensadas y objetivadas en instrumentos teóricosmetodológicos, técnicos y analíticos que se retraducen en formas particulares de percibir, apreciar y hacer investigación; funcionan en la práctica investigativa conforme a las normas de la ciencia, pero sin partir de ellas, reaccionan frente a decisiones prácticas y se manifiestan en el desarrollo empírico de una investigación y en la forma de comunicar el conocimiento construido. En ese sentido, promover su desarrollo, interiorización y recreación será una de las finalidades de la formación doctoral, donde el personal investigador, en su papel de docente, de director o directora de tesis (personal tutor) y de lector o lectora de tesis, pasan a ser mediaciones humanas que contribuyen a la formación para el oficio investigador de los sujetos doctorandos.

\section{3.- Procedimientos metodológicos}

Al pretender generar conocimiento sobre el sentido y orientación del contenido de las intervenciones que las personas lectoras académicas hacen al revisar y comunicar al estudiantado su valoración sobre los avances de investigación o borradores de la tesis, con apoyo en mediaciones discursivas, constituidas por los reportes de lecturas de avances de investigación entregados al final de su intervención oral en cada coloquio 
durante los estudios de doctorado en educación, se consideró pertinente realizar un acercamiento inductivo interpretativo al objeto de estudio.

El método implementado fue estudio de caso múltiple, el cual demanda un posicionamiento epistemológico distinto: no centrarse en lo que el caso aporta a la comprensión, sino en el fenómeno o condición presente en cada caso para ver qué es posible comprender a cerca de este (Stake, 2006). Así, el punto de partida lo constituye ese fenómeno o condición, luego se estudia cada caso focalizado en las manifestaciones de esa condición y en un segundo momento se procede a buscar similitudes y diferencias entre ellos, a fin de comprender la condición en estudio: el sentido y orientación del discurso de los lectores y lectoras como formadores en investigación en programas de posgrado. Por ello, lo relevante no solo es el individuo que realiza la función lectora, sino el contenido de su discurso, manifiesto en los reportes escritos recuperados.

El personal lector incluidos es profesorado-investigador en activo, con el grado de doctorado, adscrito al Sistema Nacional de Investigadores (SNI) del Conacyt en México, con producción académica reconocida en los campos de la educación y de las ciencias sociales, y experiencia en formación de por lo menos dos generaciones, en el nivel de doctorado. La masa textual con la que se trabajó está integrada por 100 reportes escritos de 9 de este personal, que labora en tres diferentes programas de doctorado en educación en la Región Centro-Occidente de México, y que contienen señalamientos hechos a 60 estudiantes. La recuperación de esos reportes escritos se hizo por un periodo de diez años (2003-2012).

Los reportes recuperados, en tanto producto comunicativo, contienen comentarios, observaciones y sugerencias para sus estudiantes, elaborados a partir de lo que encontraron los lectores y lectoras al revisar los avances de investigación de los sujetos doctorandos. De acuerdo con Piñuel (2002), textos como los reportes remiten a un universo de prácticas sociales y cognitivas donde cualquier interacción comunicativa cobra unos límites particulares que son mediados y mediadores de aquellas prácticas a las que sirve. En este estudio, tales prácticas se conciben en conjunto como prácticas de formación para la investigación en posgrados en educación.

Desde esta perspectiva, el análisis de esos reportes escritos se hizo con apoyo en algunos principios del análisis de contenido, el cual, de acuerdo con Piñuel (2002), consiste en un conjunto de procedimientos de interpretación de productos comunicativos (mensajes, textos o discursos) que proceden de procesos singulares de comunicación previamente registrados, y que, basados en técnicas de medida, a veces cualitativas (lógicas basadas en combinación de categorías) tienen por objeto elaborar y procesar datos relevantes sobre las condiciones mismas en que se han producido aquellos textos, o sobre las condiciones que pueden darse para su empleo posterior.

Como sostienen Taylor y Bogdan (1992), se trata de una técnica útil para formular, a partir de ciertos datos, inferencias que pueden aplicarse a un contexto; por lo mismo, se requiere abordar los datos tal y como se comunican al analista de acuerdo con el contexto en que se obtuvieron, y analizarlos en función del conocimiento que quien investigar tiene de este mismo, tal como se procedió en esta ocasión. Al respecto, McKernan (1996) señala que el análisis de contenido sugiere una orientación basada en momentos como los siguientes:

1. Definición del universo de contenido, que incluye tanto los textos como los mensajes y las formas de comunicación empleadas. En esta investigación, los textos están referidos a 100 reportes escritos de 9 lectoras y lectores académicos recuperados por un periodo de diez años, como se precisión en el punto 3.

2. Categorización de las unidades de datos, la cual puede realizarse con diversa amplitud, por ejemplo, palabras, temas, elementos, personajes, entre otros. Aquí se utilizaron frases y oraciones que comunicaban un mensaje concreto.

3. Análisis de los datos. Aunque la versión tradicional del método del análisis de contenido sugiere una cuantificación, basada en las frecuencias con las que aparecen las unidades verbales en el 
discurso aceptado por escrito, en esta investigación no se pretende trabajar solo en esa forma, aunque se dejó abierta la posibilidad de incluirla según se requiera.

Así, desde la lógica del estudio de caso múltiple, el análisis de contenido se hizo en dos niveles: a) intrasujeto o al interior del discurso recuperado de cada sujeto lector académico, b) entre sujetos, o lectura transversal del discurso entre los sujetos lectores para identificar elementos de significados sobre la orientación de la crítica en términos de lo que comunican a los doctorandos y a las doctorandas sobre la labor de la investigación y sus condiciones. A continuación, se presentan las afinidades de los nueve sujetos lectores de tesis, sobre lo que aquí se engloba bajo la categoría: pistas sobre el oficio de investigador o investigadora, producto de una lectura transversal. En ella se ubican planteamientos que, independientemente del aspecto específico de la investigación al cual hacen referencia, son una especie de tips u orientaciones relevantes, que guían al estudiantado para el desempeño satisfactorio del oficio.

Los fragmentos de discurso incluidos tienen la finalidad de mostrar, en detalle, lo que dicen y cómo lo dicen, y centrar la atención en aquellas unidades temáticas que enfatizan de manera coincidente respecto a la formación de personal investigador educativo. El código utilizado para identificar el aporte de cada lector fue L1P1E1, donde L1 refiere al número del sujeto lector, P1 número de programa y E1 número de estudiante a quien se le hacen sugerencias.

\section{4.- ANÁLisis Y Discusión DE RESUltados}

Las categorías emergentes del análisis de contenido realizado fueron las siguientes: disciplina, seriedad y compromiso; aportar conocimiento nuevo, rasgos del investigador o investigadora, la escritura académica y el uso pertinente del lenguaje, el papel de la revisión de la bibliografía, delimitar, problematizar, articulaciones, conocimiento de los métodos, función de los contextos de investigación, explicitar el involucramiento personal con el objeto, vigilancia epistemológica, y realizar afirmaciones con sustento, las cuales se desarrollan enseguida.

\section{Disciplina, seriedad y compromiso}

En el contenido de los mensajes emitidos por el personal lector de tesis en doctorado, aquí analizados, se percibe un interés porque el estudiantado comprenda que la disciplina del trabajo diario y la atención a la aportación del tutor o tutora son dos aspectos cualitativos que pueden marcar la diferencia en la formación doctoral.

En una tesis doctoral es importante producir día con día, conforme se reciben las observaciones del tutor es importante construir el documento y no redactar hasta el final con premura. L6P1E2

El señalamiento anterior se relaciona de manera directa con el sentido que tiene la actividad de investigación, la cual, según su punto de vista, involucra un alto grado de seriedad y compromiso, reflejado en la realización de un trabajo que se caracteriza por ser riguroso, claro y cuidadosamente elaborado.

Una primera impresión global de la lectura del avance es que se trata de un trabajo serio, con mucha labor de construcción personal, coherentemente expuesto y sustentado en la mayoría de los planteamientos, por lo cual es de augurar un buen trabajo en su versión final. L7P2E2 


\section{Aportar conocimiento nuevo}

Señalan con énfasis que una tesis del nivel de doctorado tiene que aportar nuevo conocimiento sobre algún campo, rasgo principal que no habrá de perder de vista el estudiantado durante su formación, como se aprecia enseguida:

Creo que en algún lugar debería destacarse con toda claridad la trascendencia de realizar esta investigación y su aportación teórica para que le quede más claro a los lectores cuál es tu aportación a la educación. L6P2E4

Un requisito de una investigación social es ser innovadora. En este y otros casos hay cierto empalmamiento o repetición de temas y objetos de estudio.... Esta situación crea un problema con respecto al requisito de innovación. L3E3R1

Esa demanda de aportar conocimiento nuevo en una tesis doctoral, según el punto de vista de los lectores o lectoras de tesis, se encuentra estrechamente asociada a la novedad de los temas que se seleccionan. Así queda expresado a continuación:

Expreso antes que nada mi felicitación a la estudiante por la selección del tema de investigación. Considero que es un tema relevante y poco estudiado y espero con interés los aportes al conocimiento que seguramente este trabajo abonará. L9P1E2

Esa insistencia sobre el aporte de conocimiento nuevo coincide con lo planteado por Deem y Brehony (2000), quienes sostienen que, un atributo fundamental de la investigación es la aportación de nuevo conocimiento. Estos mensajes también coinciden con lo señalado por Stoilescu y McDugall (2010, p.81), quienes argumentan que el estudiantado doctorando, en su condición de aprendiz del oficio de investigar, experimenta dudas acerca de lo que representa una auténtica contribución a un campo de conocimiento.

\section{Rasgos del investigador o investigadora}

En los mensajes del personal lector de tesis también se hacen presentes algunos rasgos del investigador o investigadora. Uno de ellos es el carácter creativo-constructivo que le imprime a cada una de las tareas y acciones que involucra dicha actividad, lo cual la convierte, de cierta forma, en una artesanía intelectual, donde la reflexividad se torna elemento central.

En las preguntas de investigación que planteas vale la pena reflexionar: a) las formas de acercamiento al campo, ¿te dan suficientes elementos para poder dar cuenta de cómo son los procesos formativos..., con todo lo que esta expresión implica?; b) el incorporar en la tercera pregunta juicios de valor como una asesoría sólida, un apoyo académico crucial, una motivación clara ¿te facilita o te complica la vinculación con ciertos referentes empíricos?, ¿estás dando respuesta precisa a esta pregunta al término de tu trabajo? L7P2E12

Otros rasgos enfatizados son: la seriedad y la capacidad de argumentación propia de quienes se dedican a la investigación.

No me parece conveniente el uso de un lenguaje figurado en una tesis doctoral. 'Se están asfixiando las universidades'. L6P2E4

Wikipedia es una buena enciclopedia; pero ¿es lo suficientemente seria para utilizarla en una tesis doctoral? Desde mi particular punto de vista no. L6P2E4

Otra de las características principales que habrá de desarrollar quien se forma para el oficio investigativo, según el punto de vista de las lecturas de tesis analizadas, es el dominio de su campo de conocimiento, tal como lo hace evidente una lectora en el comentario siguiente:

En el documento existe una confusión entre varias categorías que son diferentes, pero se dan como equivalentes: grupos y comunidades de investigación o epistémicas. En este sentido, falta revisar bibliografía pertinente. Los trabajos de Norma Gutiérrez sobre comunidades de investigación son un insumo importante, si lo que se quiere es trabajar sobre dichas comunidades. Existen más de treinta comunidades de investigación reconocidas en el país. Su característica es que no tienen 
una base institucional, sino que son colaboraciones interinstitucionales y que trascienden a las propias estructuras formales. Algunas se articulan con base en revistas, simposios, etc. L9P1E3

Un rasgo más referido es desarrollar la capacidad de apertura de ideas o amplitud de pensamiento, para ser capaz de mirar la complejidad del objeto. El siguiente mensaje insiste en esta idea:

Coincido en general en que la tutoría es el espacio más importante para la formación de habitus, pero no hay que cerrar la investigación a otros espacios como serían los seminarios generales o incluso los contactos que se generan por fuera del doctorado. Lo digo apoyada en una de tus conclusiones, de que el oficio de investigador es producto de la incorporación progresiva de esquemas mentales y prácticos construidos en estrecha relación con la disciplina de origen y el campo científico. Con esto no quiero decir que replantees tu trabajo, sino que vayas al trabajo de campo con un poco más de apertura. L9P1E1

Los lectores y lectoras también señalan, en sus mensajes, el sumo cuidado que habrán de tener el estudiantado doctorando con los juicios que hace, en particular, por ser un sujeto implicado en el proceso:

Me parecen un poco apresurados algunos juicios ... me pregunto si se derivan de las entrevistas hechas por Miguel o parten de su percepción ... ¿Qué mecanismos o dispositivos se han utilizado para mediar con el problema de la implicación? L9P1E1

Seriedad, disciplina, capacidad de argumentación, dominio de su campo, apertura de ideas, cuidado con los juicios que hace, son algunas características del investigador o investigadora destacadas por el personal lector, que no distan mucho de las encontradas por Deem y Brehony (2000).

\section{La escritura académica y el uso pertinente del lenguaje}

En el contenido de los mensajes que el personal lector de tesis emite, al estudiantado doctorando, se percibe una insistencia fuerte sobre la relevancia que tiene la escritura académica y el uso pertinente del lenguaje en la actividad de investigación. Respecto a la escritura académica, aparecen señalamientos sobre la función y el momento de la escritura en la investigación, donde comunican al estudiantado doctorando o investigador en formación, que habrán de desarrollar una especie de intuición para decidir el momento en que es conveniente "dar el paso" o "meter las manos", en ese arte creativo que supone escribir:

Es recomendable que la autora comience a poner énfasis en la escritura y no ya en la "exploración” (por más profunda que sea) del objeto de estudio. L8P1E3

La intuición a la que aquí se hace referencia, alude a una especie de mirada anticipada (fundamentada en lo que han leído, analizado, reflexionado, parcialmente madurado) o de consideración razonada, de que se encuentra en condiciones intelectuales, para plasmar por escrito lo hasta entonces asimilado y comprendido. Es una especie de intuición, quizá al estilo de lo que Denman y Haro (2002, p.32) designan como "intuición fundamentada".

Este señalamiento que les hacen para que se esfuercen en plasmar por escrito sus avances, coincide con lo referido por Difabio (2011, p. 941), quien expresa "la necesidad de alentar en los estudiantes el hábito de la escritura regular desde las etapas tempranas del proceso, ayudarlo a planificar la escritura, a revisarla, a establecer un horario -entre otras estrategias autorregulatorias-", como mediación clave que contribuye a generar sentimientos de logro y progreso.

El personal lector de tesis también invita al estudiantado doctorando a asumir la escritura en la actividad de investigación, como una herramienta epistémica que contribuye a clarificar el pensamiento y las ideas, a precisar el sentido y el significado de aquellas expresiones que dan forma a los enunciados que integran el contenido de un argumento, a organizar las ideas que habrán de ayudar a madurar y hacer avanzar el proyecto o reporte de investigación.

... a mí me parece que todavía hay muchos presupuestos en tu cabeza no fundamentados en datos, en antecedentes de investigación o en literatura o en datos científicos, por ejemplo, tú dices, mi experiencia, esa es de tipo personal. Aquí dices, 
el conocimiento declarativo y el procedimental se diferencian por la velocidad en que se activan iúchale!, según quién te puse yo, cuál es más rápido el que declaró o el que hizo; aquí sí te puse que haces una serie de afirmaciones muy categóricas que cuesta trabajo aplicar en tu propio discurso. L1P2E4

Ya señala Carlino (2006, p. 8) que "escribir es un método para pensar y no solo un canal de comunicación... la escritura permite volver sobre lo pensado tantas veces como sea necesario” (p. 10), y que en la investigación se requiere escribir “antes, durante y después” (p.11).

Otro elemento referido de manera constante es la necesidad de que el estudiantado investigador en formación desarrolle un estilo de escritura académica orientado hacia la difusión del conocimiento, que incorpore las convenciones específicas de cada disciplina o campo de conocimiento, y respete las pautas o normas de escritura establecidas por la comunidad científica para tal propósito, como se aprecia enseguida.

En general hay muchos problemas para saber citar, para saber conservar o respetar las formas tradicionales del manejo de un texto académico y cuidar la redacción formal. L1P2E2

Utilizar términos claros, científicos, ya que los coloquiales, no se entenderán en los diferentes contextos. L4P2E4

Revisar brincos conceptuales entre párrafos e ideas. L4P2E3

Se trata de un modo de escritura, cuya estructura organizativa refleje un alto nivel de rigor y formalidad; dé cuenta del dominio formal del lenguaje escrito; el uso correcto de las reglas gramaticales y sintácticas; y dé cuenta también de que el estudiantado investigador en formación se ha transformado en un usuario competente de la lengua escrita.

Como se ha señalado en párrafos anteriores, con base en los comentarios, la escritura, en la actividad de investigación, requiere un uso preciso del lenguaje que deje escasos márgenes para la imprecisión, la ambigüedad o la atribución de sentido y significado escasamente pertinentes, que conduzcan a una fuente de errores diversos, por ejemplo, de orden teórico-conceptual, teórico-metodológico, epistemológico y de análisis de datos, entre otros. En ese sentido, el personal lector es enfático en la construcción de un discurso que se oriente de manera clara, definida y suficiente, hacia lo que es el foco de la investigación, congruente con la intención de cada momento de la investigación.

En una reconstrucción de esa parte (de problematización y construcción del objeto de estudio) convendría situar actores sociales, contenidos cuyo interés es central a partir de las categorías del análisis y, al mismo tiempo, ir configurando las variables que interesan para esta tesis. Ir más allá de citar problemas y proponer, en cambio, lo que es más relevante estudiar y que aparecerá como constante en la formulación discursiva de la tesis. L5P1E3

De manera paralela, se hace notar que ese uso pertinente del lenguaje abarca el dominio de los asuntos sintáctico-gramaticales, ortográficos, de citar y referenciar la bibliografía consultada con precisión, entre otros, los cuales son señalados como observaciones de forma, donde se suelen expresar calificativos de "indigno", sobre aquellos textos que presentan deficiencias en la expresión escrita, puesto que les resulta inadmisible que alguien llegue al doctorado sin haber logrado mejores formas de expresión, redacción y ortografía, como se aprecia a continuación:

La presentación del documento vuelve a tener múltiples deficiencias en cuanto a redacción y ortografía, indignas de un trabajo de nivel de posgrado. L8P1E2

En lo inmediato, creo que el nivel de presentación formal del borrador es todavía muy insatisfactorio ... A todo lo largo del texto es indispensable una extensa y detallada corrección de ortografía, digitación y redacción, pues el descuido en el uso del lenguaje es imperdonable en cualquier trabajo académico, pero más en uno que trata principalmente el análisis del lenguaje mismo. L8P1E4

Es necesario también, en el plano formal, revisar que todas las referencias bibliográficas estén completas y correctas. Es decir, que en las citas se señalen los números de página; que no haya obras referidas que no estén en la bibliografía ni viceversa; que todo fragmento textual tomado de otras fuentes esté debidamente referido. Esto es especialmente importante en el marco teórico, pero debe aplicarse en todo el texto. L8P1E4

Estos señalamientos son coincidentes con lo expresado por Dietz, Jansen y Wadee (2006, p.40), quienes argumentan que "el cuidado lingüístico es un prerrequisito para la escritura académica", donde, además, 
como lo señalan Rodenes, Chismol y Arango (2000, p. 474), se requiere dar orden y coherencia a un trabajo intelectual de gran envergadura y poco estructurado, como lo es la actividad de investigación.

\section{El papel de la revisión de la bibliografía}

Otro elemento en el que los mensajes del personal lector de tesis se focalizan es en la revisión bibliográfica, como tarea permanente en la actividad de investigación, la cual involucra un arduo y amplio trabajo de lectura que posibilite contar con una diversidad de aportes para construir una visión amplia y profunda sobre cierto campo temático, y sobre cierto objeto de estudio en particular, como se lee en los comentarios siguientes:

La bibliografía es muy poca para una tesis doctoral, hay que completarla porque seguro que consultaste muchas más fuentes que las enlistadas. L7P2E14

En el estado de conocimiento está más ampliamente revisado lo de género que lo de redes sociales, hay que ampliar esa revisión, pero también es importante no construir básicamente sobre una misma autora. L7P1E7

Como lo hacen, esa revisión bibliográfica sirve de base para generar ideas y percibir por dónde continuar el avance de la investigación, pero también apoya de manera central la fundamentación del estudio, en cuyos señalamientos se perciben algunas pistas para ello:

En el documento presentado se distinguen dos partes: primero los ajustes de la fundamentación del estudio y segundo los materiales empíricos sobre los que se ha trabajado. Es una buena decisión el estructurar el documento como un avance (primer borrador) de tesis, si bien no puede todavía juzgarse como tal. Hay todavía mucho trabajo por realizar para adquirir la mínima precisión requerida para la presentación de los fundamentos y contextos del trabajo en el nivel de una tesis de doctorado. Es necesario avanzar en el logro de la consistencia lógica y en la formulación discursiva más adecuada de los diversos elementos que en distintos niveles articulados han de construir la fundamentación. L8P1E4

De manera asociada a esa fundamentación del estudio, producto de una amplia revisión bibliográfica, llaman la atención del estudiantado para que revise la coherencia en sus planteamientos y el uso correcto de los conceptos, como se aprecia enseguida:

Página 9 dices 'a propósito de las experiencias que derivan en situaciones de fracaso escolar, en el marco de sus contextos locales de actuación, como la escuela, el salón de clases y la familia.' En la hipótesis se dice que solo interesa el aprendizaje en el aula. ¿Entonces por qué haces referencia a estos tres contextos? L6P1E1

Pág. 6, párrafo 3, dice que la epistemología es una disciplina y una ciencia. ¿Entonces qué es disciplina o ciencia? ¿No es lo mismo? L6P1E3

También se percibe que muestran cierta insistencia para que el estudiantado doctorando desarrolle un fuerte trabajo teórico-conceptual, con base en esa amplia revisión bibliográfica que realiza, el cual permeará todo el trabajo de investigación, dará orientación a las preguntas planteadas, y servirá de base para la comprensión del objeto de estudio y la construcción de explicaciones plausibles al problema planteado.

En el capítulo II se presentan los conceptos clave del estudio sin llegar a conceptuar aquellos que directamente implican a los de las preguntas de investigación. ... Se presenta un inicio de conceptualización desde los diccionarios, situación que no es necesario incluirla en un trabajo doctoral, sobre todo porque no se llega a conceptuar desde el investigador. L4P2E6

En el apartado teórico, considero que se deben revisar los conceptos clave y centrarnos en ellos, quitar la información que solo es para conocimiento de la autora. L4P2E2

Un señalamiento fundamental que realizan es que, si bien la actividad de investigación se nutre de la revisión de fuentes documentales y la incorporación de ciertos referentes teóricos-conceptuales, a propósito de cierto objeto de estudio, la tarea principal de quien investiga consiste en aprender a utilizar la teoría para pensar con apoyo en ella, y no solo a trasladarla de manera descriptiva en la investigación que desarrolla.

Me parece que abusas en transcribir fichas de literatura que lees, no a usarlas, hay muchos fragmentos reseñados y eso tiene dos problemas, uno que me parece no logras decir y eso que dice Pozo para qué me sirve en mi objeto de estudio, en qué me 
va a beneficiar o apoyar en la pregunta de investigación para que yo pueda usar esas explicaciones, ... entonces, me parece que no es un buen procedimiento para una tesis de doctorado. L1P2E4

Aprendí que, si uno no se da cuenta de que solamente está trasladando la teoría, entonces nunca logra establecer ese puente entre pregunta, teoría y lo que supongo voy a encontrar. L1P2E4

Del señalamiento anterior se desprende que aprender a pensar con apoyo en la teoría en la actividad de investigación es un elemento clave que posibilita que quien investiga logre construir argumentos personales sólidos, con voz propia como autor o autora de ideas.

\section{Delimitar}

De manera paralela, el personal lector de tesis refiere, en sus mensajes, que, en ese proceso de construcción conceptual del objeto, es importante reflexionar sobre la amplitud, el alcance, y las implicaciones prácticas y metodológicas de eso que se argumenta, para entonces estar en condiciones de delimitar de manera clara y precisa, conceptos, objeto de estudio, preguntas de investigación, universos de estudio, observables, entre otros, como acción constante que habrá de realizar el sujeto investigador durante la generación de conocimiento.

Cuando estás trabajando las dimensiones, yo creo que en la dimensión socio-histórica vale la pena que hagas una delimitación, porque estás hablando de reconstruir el contexto y además en dos países, entonces hay que ir acotando. L2P3E2

Habría que acotar y delimitar el objeto de estudio y el universo de investigación. L2P1E3

Se deben delimitar bien las preguntas de investigación. L2P1E5

Falta delimitar la dimensión temporal: ¿qué aporta o qué límites presenta la gestión foxista para el logro de la educación de calidad que incluye al desarrollo sustentable? L5P3E6

Los señalamientos anteriores tienen que ver con esa condición necesaria que todo sujeto investigador requiere desarrollar para abordar objetos de estudios asibles, acotados. Como señala Wainerman (2001, p. 49), "cuanto menor la experiencia [en investigación] más difícil es aceptar que no se puede estudiar todo ("el mundo y sus contornos"...) y que hay que acotar; también que la que uno planea no es la última investigación de la vida...".

De manera paralela a la actividad de delimitar en la investigación, les insisten, en sus mensajes, en tener el cuidado suficiente de no caer en rodeos ni reduccionismos, como se ilustra a continuación:

Aunque hay una acotación temporal contemporánea, la propuesta metodológica de hacer toda una caracterización de la Economía Política de cada uno de los sexenios desde los 40 's, puede llevar a hacer un rodeo innecesario que abulte la tesis con generalidades que no le aportan gran cosa al estudio de la política educativa hacia la universidad pública. L5P1E2

Hay una cierta simplificación del estado de las ciencias sociales en general y en particular en México, lo cual limita la comprensión del autor sobre la contribución que podrían hacer ciertos estudios al tema de esta tesis. Partir de una calificación adjetivada de la producción científica social, no conduce a establecer una nueva visión de la ciencia como la que el autor pretende. Se necesita un trabajo de discusión de mayor profundidad en este sentido. L5P1E1

Parece ser que estos señalamientos se orientan a hacer notar al estudiantado doctorando, el sumo cuidado que habrá de tener con cada una de las acciones cognitivas que despliega durante la investigación, y la incidencia que puede tener cada una de ellas en la generación del conocimiento.

\section{Problematizar}

En relación estrecha con los señalamientos anteriores, el personal lector de tesis hace notar al estudiantado doctorando que el oficio de investigar no puede ser desarrollado con la calidad y el rigor científico requerido, si se carece de un fuerte trabajo de problematización, esto es, un serio esfuerzo de cuestionamiento, crítica, 
reflexividad, desestabilización, crisis, ruptura y reconstrucción de los planteamientos emergidos a propósito de cierto objeto de estudio.

\begin{abstract}
Al presentar los problemas, se deben problematizar, es decir, preguntarse por qué existe, cuál es la razón. Además, explicar qué significa el problema y relacionar los problemas y tener claro su orden con una lógica de la misma índole, no mezclar problemas que no tienen relación. Describir cómo se observan los problemas en el campo donde se desarrollan, al final de la explicación hacer preguntas y conclusiones. L4P2E4

No queda establecido un espacio de problematización, lo cual deja ver que la investigación no surge de una necesidad real (aparentemente), es decir, no queda claramente establecida la problematización de donde surge la necesidad de hacer este estudio. L4P2E6
\end{abstract}

Los señalamientos anteriores tienen que ver con esa actitud particular que habrá de desarrollar toda persona que investiga: no dar por hecho algo, justamente porque su labor es construir preguntas, plantear explicaciones tentativas o hipótesis, y de manera asociada a un proceso de razonamiento, tratar de dar respuestas.

$\mathrm{Al}$ interior de esta actividad de problematización, llaman la atención del estudiantado a caer en la cuenta de ciertas confusiones entre posturas reaccionarias y posturas académicas, las cuales deberán cuidar. Si bien la etapa de problematización suele darse a partir de situaciones que cuestionan a quien investiga, y ante las cuales es posible que reaccionen con diversas emociones, el proceso de transformación de esas reacciones iniciales en intereses académicos resulta fundamental.

Leo un sentido de defensa, o reacción, como tú lo dijiste ahorita, de una postura reaccionaria. Es una reacción de resistencia
ante una imposición del sistema que no capta la lógica del sistema económico ni se basa en una lógica propia. Entonces es,
simplemente una resistencia que está condenada a reventar. Muy probablemente lo que reviente sea el sistema, como que eso
sí está bien previsto, pero que los objetivos digamos de la evaluación, cualquiera que sea, o de la educación, en ese sistema,
pues aparecen difusos, como muy frecuentemente sucede. L8P3E5
Se puede mantener una postura muy crítica al respecto, muy rebelde, digamos, pero creo que hay que construir en términos
mucho más fríos el objeto de investigación para caminar en la dirección de una oposición más sólida a este tipo de usos de
una racionalidad en la que uno puede no estar de acuerdo. L8P3E5

Desde comentarios como los anteriores, el personal lector de tesis, en tanto grupo formador, contribuye a que el estudiantado distinga entre posturas reaccionarias -que pueden ser legítimas y que no están reñidas con el pensamiento crítico que caracteriza la labor investigativa-, y posturas académicas, desde las cuales se responde, transformando las primeras inquietudes o reacciones, en motivos para plantear sólidas preguntas de investigación que generen conocimiento.

En este mismo orden de ideas, en los mensajes se aprecia un especial énfasis de llamado a la cautela de quien investiga en todo aquello que afirma y plasma en cualquier forma del lenguaje, así se lee en los señalamientos siguientes:

No es posible generalizar, a partir del estudio de situaciones particulares muy concretas, la dinámica del campo científico y del educativo; corresponden en todo caso a procesos específicos en instituciones concretas. L2P1E3

Cuidar la postura normativa que es posible advertir en varios párrafos del trabajo. L2P3E3

Hay que matizar la afirmación de que la enseñanza del habitus científico está asociada a procesos específicos de formación vía posgrado, ya que no siempre es así. L2P1E3

El conjunto de estos comentarios del personal lector de tesis se orienta, en forma insistente, a quienes se inician en el oficio, a caer en la cuenta de puntos álgidos del trabajo de investigación, donde se requiere, entre otras cosas, argumentar, delimitar, comprender el sentido de lo que se afirma, en la necesidad de una mayor precisión, y profundización gradual, de los elementos del proyecto de investigación que se lleva a cabo.

El nivel de la construcción del proyecto de investigación presenta algunos avances, pero sigue dejando pendientes tanto la profundización y ajuste de sus diversas partes como la articulación general. Habrá que recuperar tiempo durante el semestre para buscar la mayor consistencia en el planteamiento, y mucha mayor densidad en su fundamentación y desarrollo. L8P1E1 
Me da la impresión de que es un acercamiento a una zona muy peligrosa. Igual hay que tomar algunas precauciones que no sé si las tenga previstas. L8P1E5

Estas son recomendaciones que ilustran cómo, aquellos acercamientos sucesivos en los que se cuida cada vez más la precisión y la profundidad son los que abren paso a una construcción teórica-conceptual, y metodológica sólida y pertinente en una investigación.

Otro elemento al que hacen referencia, y que es considerado como un reto al que se enfrenta de manera cotidiana todo sujeto investigador, es el de darse cuenta de a dónde lo conduce lo que está construyendo, y si necesita reorientar o reformular en un momento dado sus construcciones, tal como se aprecia a continuación:

La transformación se toma como un presupuesto, cuando la investigación científica requiere de plantear preguntas e hipótesis que se responderán. Sin embargo, a nivel metodológico el razonamiento se da a posteriori. L3 P3E1

El señalamiento anterior tiene que ver con la demanda de que el sujeto investigador en formación desarrolle habilidades de orden metacognitivo, como las referidas por Moreno (2002) en su perfil de habilidades investigativas.

\section{Articulaciones}

Uno de los mensajes que el personal lector de tesis emite con insistencia, al estudiantado, es que la generación de conocimiento por vía de la investigación, requiere del desarrollo de un pensamiento relacional, una visión integral, a manera de un todo unificado de aquellas tareas y acciones que dan forma a una investigación, donde se asuma que la realización de una tarea o actividad tiene incidencia y repercusiones directas en las actividades y tareas siguientes, y en sus resultados: el conocimiento generado. Así se aprecia a continuación:

Da la impresión de que en el referente teórico faltan algunos elementos que más adelante permitan justificar las formas de análisis de la producción escrita de los estudiantes de la maestría, ¿o solo se trataba de hacer el análisis en forma deductiva conforme a un modelo ya establecido, por ejemplo, el de Van Dijk? L7P2E11

La hipótesis es congruente con el problema planteado, aunque lo que se realiza parece que no aportará elementos suficientes para contrastarla, o por lo menos no queda claro que los datos recabados podrán permitir afirmar algo de esta naturaleza. L7P1E4

Paralelamente al señalamiento anterior, otra demanda constante que solicitan es que, de manera asociada al desarrollo de ese pensamiento relacional, logren alcanzar y sostener un alto grado de articulación interna entre los diversos elementos presentes en la investigación que realizan, por ejemplo, entre preguntas de investigación, objetivos, hipótesis, perspectiva teórica construida, método de investigación y de análisis de los datos asumidos, entre otros, como sistema o todo articulado, según se aprecia en los comentarios siguientes:

Relacionar pregunta-objetivos-evidencias-hallazgos. L4P2E4

Creo que esto también es muy importante, que el análisis tiene que estar muy, muy vinculado con un marco conceptual sólidamente trabajado y que todavía está débil, todavía no termina de cuajar ... también me preocupa que las entrevistas estuvieran despegadas de un marco teórico-conceptual y metodológico más profundo, porque luego a la hora que tengas que redactar y que tengas que vincular el análisis con tu perspectiva teórica te va a dar mucho trabajo, vas a tener que forzar los datos a la parte conceptual. L1P2E2

Esta insistencia de los lectores sobre el sentido de articulación, de coherencia entre lo que el estudiantado va produciendo, es compartida por Sautu (2001, p. 227) cuando expresa: "como investigadora por toda mi vida a tiempo completo, he conocido el enorme esfuerzo que significa poner juntos todos los elementos de un proyecto de investigación". 


\section{Conocimiento de los métodos}

El personal lector señala, al estudiantado doctorando, la importancia que tiene, en la actividad de investigación, lograr un conocimiento amplio y profundo de la naturaleza y función de la teoría, los métodos, las técnicas e instrumentos de recolección de información existentes, y reconocidos como pertinentes por la comunidad científica.

La tesis del estudiante tiene serias deficiencias estructurales, como es tomar una teoría que no es coincidente con el método y la técnica utilizada en el desarrollo de la investigación. La teoría asumida no es afín con la técnica utilizada para medir comprensión lectora. ... no entiendo cómo esta visión del lector activo puede empatar con una donde se le exige recordar la palabra exacta escrita en lo que acaba de leer. L1P3E5

Habría que considerar que el método de investigación-acción requiere involucrar en las actividades y decisiones a la comunidad de forma integral y democrática. En este caso, se ha limitado el concepto de comunidad a las personas que voluntariamente asisten al parque y participan en los talleres. ¿Es esta la forma más apropiada de definir una comunidad de un proyecto de investigación-acción? Esta es una pregunta que te serviría para considerar cómo atraer más personas o para acercarte más a la comunidad en general. L3P3E6

En particular, sus mensajes insisten en que el estudiantado logre un conocimiento extendido de los métodos, lo cual incluye, entre otras cosas, poder apreciar las condiciones en las que es posible utilizar cada uno de ellos, según su naturaleza epistémica.

Otro aspecto importante referido en sus mensajes es la necesidad de argumentar o justificar las decisiones que se toman en todos los momentos del proceso de investigación, tal como se lee enseguida:

La primera pregunta que te haría es ¿cómo justificar esta investigación dentro de un doctorado en educación? Es decir, que tú buscaras la forma de argumentar cuáles serían los aportes, cuál la dimensión educativa de tu estudio. L2P1E2

El señalamiento anterior parece ser que se orienta a que el estudiantado comprenda que la actividad de investigación involucra, de principio a fin, la toma de decisiones diversas, pero que estas no son tomadas como una ocurrencia de momento, y de manera arbitraria y "por debajo de la mesa", sino que requieren ser justificadas y argumentadas con fundamentos.

\section{Función de los contextos de investigación}

En los mensajes del personal lector se enuncian señalamientos sobre la función de los contextos, considerados como elemento clave para apoyar la comprensión, no solo de la naturaleza del objeto de estudio con el que se está trabajando, sino también del posible alcance de las aportaciones que se generarán en una investigación específica, o de la evolución (histórica, social, política) que ha caracterizado el entorno en el que se investiga un problema particular, como se aprecia a continuación:

\footnotetext{
Hay un apartado que inicia reconstruyendo la historia de la investigación educativa y su incorporación en las instituciones de educación superior, pero se desconoce todo el contexto socio-político a través del cual es posible entender por qué se incluye la investigación como una prioridad y práctica privilegiada en las IES, por qué las razones de su institucionalización y expansión. Es importante la revisión de políticas públicas y educativas a partir de los 70 para entender en qué forma impactan, tanto en el contexto nacional como en el internacional. L2P1E3

Si vas a trabajar un oficio o una profesión, ese elemento de la constitución misma de la profesión y la evolución histórica y social de dicha profesión va repercutiendo también en el tipo de prácticas, entonces es fundamental indagar cuáles son las características de este oficio o profesión, cómo se estructura, cómo se difunde en otros países. L2P1E2
}

De los señalamientos anteriores, se desprende invitan al estudiantado a comprender el sentido de los contextos en dos direcciones: una que podría llamarse micro, en tanto que el sujeto investigador habrá de ubicar claramente el contexto en el que surge y se desarrolla una investigación; y una dirección macro, cuyo énfasis está en el alcance de las afirmaciones (conclusiones) que se pueden hacer al término de una 
investigación, tomando en cuenta las dimensiones del objeto de estudio y las características del universo al que estuvo referido el problema de investigación (Moreno, Torres y Jiménez, 2014).

Esa dirección macro a la que hacen referencia es congruente con el planteamiento de Ratcliffe y González del Valle (2000, p. 61), en el sentido de que "no hay leyes o verdades universales, sino leyes contextualmente determinadas".

\section{Explicitar el involucramiento personal con el objeto}

Un elemento que refiere de manera constante el personal lector, por encontrarlo ausente con frecuencia en los avances de tesis de doctorado, es la necesidad de explicitar el involucramiento personal que la persona investigadora tiene con su objeto de estudio, y la conveniencia de hacerlo desde el momento en que conforma un "espacio de aparición" para su interés por una temática, muchas de las veces en forma vinculada con la propia experiencia:

El tesista no explicita su experiencia previa en el tema, que sí la tiene, en este apartado. Ello dificulta entender la manera en que asume su investigación doctoral como proceso personal. L5P1E1

$\mathrm{Al}$ respecto, Moreno (2002) sostiene que, como parte de las habilidades para investigar, se tiene que ser capaz de "objetivar el involucramiento con lo que se estudia", entendido esto, como el hecho de que el sujeto que investiga sepa dar cuenta de sus intereses y su vinculación con el objeto.

Otra invitación que hace el personal lector al estudiantado, de manera paralela a la anterior, es que aprendan a tomar distancia y buscar imparcialidad respecto de los datos que obtienen de ciertos contextos y sujetos:

Creo que será mucho más fácil ver esos factores analíticamente si tomas esa distancia institucional. L3P2E2

Este es un problema de reactividad que toda investigación con sujetos humanos debe abordar; sin embargo, los procedimientos rigurosos de investigación nos requieren la capacidad de una evaluación imparcial, objetiva de la realidad humana y social. El investigador debe escuchar a sus sujetos, pero también debe digerir sus discursos y autoreportes, cuestionarlos de una forma crítica para realizar una buena investigación cualitativa. L3P3E4

Esa capacidad de "mirar desde fuera" algo de lo que la persona misma forma parte, es una característica que ha sido desarrollada conceptualmente desde perspectivas de investigación cualitativas, particularmente, con fines de búsqueda de datos en lo cotidiano, por ejemplo, Denman y Haro (2002, p. 42) aluden a la "doble actitud metodológica" que quien investiga habrá de cultivar, y que le permite, por una parte, identificarse lo suficiente con sus sujetos de estudio para comprender sus motivos y, por otra, mantener una actitud emocionalmente neutral y objetiva, en bien de la objetividad de sus conclusiones.

\section{Vigilancia epistemológica}

Otra insistencia recurrente que hace el personal lector de tesis, al estudiantado doctorando, es ir más allá del deber ser, de la descripción, del seguimiento de instrucciones, para romper con ciertos esquemas preestablecidos y desarrollar mayor autonomía, que posibilite pasar al nivel constructivo-interpretativo que caracteriza toda investigación.

La tesista tiene ya la mayor parte de los elementos, pero creo que todavía viene arrastrando algunos elementos de los que no se ha desprendido y pienso en particular en su manera de abordar la construcción del problema. Percibo que todavía le pesa mucho el "deber ser", es decir, ella tiene sus preocupaciones de cómo convivir juntos, de qué puede costar la educación, de por dónde nos puede ir mejor, de cómo integrar el todo, pero eso no le ha permitido entrar a la investigación ya fina y hacer un diagnóstico de qué se está intentando para poder lograr todos esos buenos propósitos que tiene. L5P1E6 
Voy a decir algo que quisiera que quedara como anecdótico, y es que la tesista es extremadamente trabajadora, pero me hace demasiado caso, entonces yo creo que hay que tomar distancia de su tutor y de sus lectores y reforzar sus propios puntos de vista para que al comprometerse con ellos pueda irse más al fondo de la cuestión. L5P1E6

No hay que permanecer en un estudio descriptivo, como la tesista lo afirma, pues un doctorado supone una investigación original que trasciende la mera descripción. L5P1E6

De manera paralela, invitan al estudiantado a desarrollar el trabajo de análisis de los datos con un alto grado de rigor y vigilancia epistemológica sobre las interpretaciones e inferencias que realizan, con base en el material empírico recabado, donde, las afirmaciones (teorizaciones) realizadas como explicaciones tentativas del problema planteado sean sostenibles.

Considero importante retomar en las descripciones de lo que hicieron los profesores, sus propios decires y no incorporar "proposiciones" que en su momento dicen lo mismo que la viñeta. Valorar dicha posibilidad. De igual manera fortalecer el trabajo en la parte interpretativa con referentes teóricos que enriquezcan la presentación del documento y mejoren su redacción y no se perciban los planteamientos de manera tan repetitiva. L 4P2E2

La interpretación es un diálogo que nos lleva a entrelazar al sujeto de la investigación, al contexto donde éste se desenvuelve y el conocimiento que se generó o se produce, ya que se convierte en el andamiaje argumental producto de la investigación realizada, razón por la cual el quedarse en el plano de la congruencia entre ellos no genera aporte al campo de conocimiento. L4P2E3

Los señalamientos anteriores permiten apreciar que el trabajo de análisis e interpretación del dato es uno de los momentos más álgidos en la actividad de investigación, no solo por el sumo cuidado que dicha tarea requiere, sino porque es un momento fuerte de "batalla" con el dato y de construcción con base en el dato, pero de manera articulada con los elementos teóricos-conceptuales trabajados previamente, para, entonces, llegar a construir explicaciones plausibles con un alto grado de credibilidad.

\section{Realizar afirmaciones con sustento}

Un elemento clave en el que insiste el personal lector de tesis en sus mensajes es que, durante el desarrollo del análisis e interpretación de los datos, quien investiga realice inferencias y aseveraciones de tipo teórico, con base en el material empírico recabado, cuidando no caer en interpretaciones fallidas o sobreinterpretaciones.

Aquí te puse que no hay que inferir posiciones teóricas-epistemológicas porque el profesor diga que es tal, ¿̨lo viste actuar en consecuencia?, ¿verdad que no? Pues yo no puedo inferir posiciones, ahí el análisis falla. ... Cuidado, se infiere en tu análisis que el docente no planea sus actividades áulicas, sin embargo, sí tiene idea de cómo trabajar por tema. Y ¿cómo sabes que no planeó?, ¿le preguntaste eso?, ¿no?, entonces yo no puedo juzgar. Cuidado con etiquetar juicios de valor. L1P2E1

Las conclusiones generales no están suficientemente fundamentadas y algunas no son conclusiones. En general el trabajo debe ser replanteado por lo que no es posible considerarlo una tesis de nivel doctoral. L1P1E5

Dicha actividad, según el punto de vista del personal lector, involucra cuidar el alcance o las implicaciones de tales afirmaciones, o en todo caso, aprender a relativizar (matizar) aquellas que contienen en sí mismas un carácter radical de afirmación.

Hay que hacer un cuidadoso análisis de lo que se afirma y de si hay suficiente sustento para afirmarlo. Pulir el trabajo en ese sentido es lo principal. L7P2E1

En la página 9 hay una aseveración radical que convendría relativizar: "en algunos casos los rectores no saben nada de la vida académica ni de la institución en sí". L7P1E3

Advierten al estudiantado doctorando sobre formas impropias de realizar afirmaciones en un trabajo de investigación, en particular, cuando se cae en la vaguedad por falta de elementos, como se aprecia a continuación:

Se hacen afirmaciones que no se sustentan en los resultados ni en la bibliografía, que desde mi punto de vista en un trabajo de investigación de doctorado no deben aparecer, como 'la apreciación es pobre', o 'no dice mucho'. L6P2E4 
El conjunto de estas pistas sobre el oficio de investigar permite percibir que la generación de conocimiento involucra diversidad de tareas y acciones que requieren ser desarrolladas con rigor científico. En ese sentido, este grupo lector de tesis, contribuye con sus comentarios a conformar una visión de la investigación como la que sostienen Denman y Haro (2002, p. 47), cuando afirman que el rigor científico ha de referirse a la calidad de las decisiones que se efectúan en el proceso de investigación, visto "como un todo en el cual cada fase está en interacción dinámica con las otras y con la totalidad del mismo proceso", en concordancia con esa imagen de la espiral, donde cada etapa se apoya y alimenta de las demás.

Sutilezas o tips como los anteriores pasan a formar parte de lo que aquí se considera pistas sobre el oficio de la persona investigadora, las cuales distan mucho de ser una prescripción sobre cómo realizar o reportar investigación. Se trata de saberes sustentados en la experiencia investigativa, que solo se despliegan en la socialización del oficio al lado de un sujeto experto en dicha actividad, por tanto, se encuentran más allá de lo referido en los manuales de metodología de la investigación; se asoman a los implícitos de la investigación, y contribuyen a que el estudiantado doctorando empiece a vislumbrar ese "algo no codificable, difícil de transmitir del oficio de investigador" (Wainerman, 2001, p. 21).

\section{5.- A MANERA DE CIERRE}

De cierta forma, este conjunto de mensajes enviados por el personal lector, al estudiantado doctorando, son considerados como pistas sobre el oficio de investigar, en tanto contribuyen a comprender la artesanía intelectual que demanda esta actividad, así como el sentido de cada uno de los elementos y procesos que conforman una investigación, y su respectiva estructuración en un todo articulado, como rasgos clave que habrá de caracterizar una investigación de buena calidad.

En dichos mensajes se advierten riesgos que hay que prever, como por ejemplo, hacer afirmaciones categóricas sin sustento y generalizar sin fundamento; cuestiones que hay que evitar; aspectos en los que hay que tener suma cautela, como sustentar todo lo que se afirma en antecedentes de investigación, en referentes teóricos o empíricos; no caer en rodeos ni en reduccionismos en el tratamiento de las temáticas; cuidar el rigor académico, incluso, en el tipo de lenguaje que se usa y en las fuentes que se consultan. Hay recomendaciones que pretenden dinamizar la comprensión de lo que supone investigar, como la necesidad de vigilancia epistemológica a lo largo del proceso de investigación; equilibrio entre descripción, análisis e interpretación; invitación a la ruptura de esquemas, como elemento necesario de evolución de las habilidades investigativas. Todo ello como elementos disposicionales que dan forma relativa al habitus científico (Torres, 2013), cuyo dominio pasa a ser el derecho de entrada al campo.

Es decir, el personal lector académico aporta elementos para que el estudiantado doctorando construya una visión de la investigación que se caracteriza por ser rigurosa, donde los aspectos epistemológicos pasan a ser centrales, como los referidos en las categorías de aportar conocimiento nuevo, rasgos de quien investiga, escritura académica y uso pertinente de lenguaje, problematización, articulaciones, vigilancia epistemológica y realizar afirmaciones con sustento.

Este conjunto de rasgos, emitidos por voces calificadas del campo para hablar con autoridad científica sobre este quehacer, en cierto grado son reflejo del deber ser sobre la investigación que han interiorizado en el transcurso de su formación y desarrollo como investigadores e investigadoras. Se trata de un deber ser que se aprende haciendo en la práctica investigativa, por lo tanto, es insuficiente que el estudiantado las repita fielmente para asumir que ha aprendido a investigar. El reto está en que la producción investigativa, que ponen a consideración del personal lector, alcance las características recomendadas, una vez que dan atención crítica a sus sugerencias.

La cuestión es, si toda expresión de deber ser ha de ser considerada como un simple manual de investigación, y si los mensajes de los lectores y lectoras son mera expresión de una normativa que puede orientar la producción de investigación. Aquí es donde se sostiene, con base en el análisis realizado, que la función 
lectora de tesis, en tanto formativa, va más allá de ser una voz normativa, como si se tratara de un manual de investigación. Sus intervenciones proporcionan evidencias concretas, para ser consideradas como una verdadera mediación pedagógica en la formación investigativa, no siempre valorada en su justa dimensión, porque también existen casos que realizan esta función "a la ligera”, la usan para desvalorar a los estudiantes o para criticar a sus pares por otro tipo de motivos, como las posiciones que se juegan en la estructura de poder en un campo científico como la educación.

Por ello, es importante que la figura de la lectora o lector académico sea revalorada en el marco de los estudios de posgrado, pero también sea asumida con mayor seriedad y compromiso por quienes la realizan. A nivel institucional, esto implica asumir criterios claves para su selección, como, por ejemplo, la experiencia de investigación, su conocimiento en la temática que se aborda, así como su disposición para formar a otras personas en la actividad de investigación.

\section{ReFERENCIAS}

Bourdieu, P. (1976). Le champ scientifique [El campo científico]. Actes de la Recherche en Sciences Sociales, 2(2), 88-104. doi: https://doi.org/10.3406/arss.1976.3454

Bourdieu, P. (2003). El oficio de científico. Ciencia de la ciencia y la reflexividad. Curso del Collège de France 2000-2001. Barcelona: ANAGRAMA.

Carlino, P. (2006). La escritura en la investigación. En C.Wainerman (Dir.), Documento de trabajo N.o 19, (pp. 4-43). Buenos Aires, Argentina: Universidad de San Andrés.

Deem, R. y Brehony, K. (2000). Doctoral students' access to research cultures: are some more unequal than others? [El acceso de los estudiantes de doctorado a culturas de investigación: ¿Son algunos más desiguales que otros?] Studies in higher education, 2(25), 149-165. doi: https://doi.org/10.1080/713696138

Denman, C. y Haro, J. (2002). Introducción: Trayectoria y desvaríos de los métodos cualitativos en la investigación social. En C. Denman, y J. Haro (Coords.), Por los rincones. Antología de métodos cualitativos en la investigación social (pp. 9-48). Hermosillo, Sonora, México: El Colegio de Sonora.

Dietz, A. J., Jansen, J. y Wadee, A. (2006). Effective PhD Supervision and Mentorship. A workbook based on experiences from South Africa and Netherlands [Doctorado eficaz supervisión y tutoría. Un libro basado en las experiencias de Sudáfrica y Países Bajos]. Amsterdam: Rozemberg-UNISA Press.

Difabio, H. (2011). Las funciones del tutor de la tesis doctoral en Educación. Revista Mexicana de Investigación Educativa, 50(16), 935-959.

Holbrook, A., Bourke, S., Lovat, T. y Fairbairn, H. (2008). Consistency and inconsistency in $\mathrm{PhD}$ thesis examination [Coherencia e incoherencia en el examen de tesis doctoral]. Australian Journal of Education, 1(52), 36-48. doi: https://doi.org/10.1177/000494410805200103

Jack, B. (2002). The final hurdle: preparation for the $\mathrm{PhD}$ viva examination [El último obstáculo: La preparación para el examen de doctorado en vivo]. Nurse Researcher, 2(10), 66-76.

McKernan, J. (1996). Investigación-acción y currículum. Madrid: Morata.

Moreno-Bayardo, M. G., Torres, J., Jiménez, J. M. (2013). Los lectores académicos como formadores de investigadores en el posgrado. Retrato de un estilo de formación, en Memorias del XII Congreso Nacional de Investigación Educativa, Guanajuato México, Recuperado de http://www.comie.org.mx/congreso/memoriaelectronica/v12/ doc/0080.pdf?cv=1

Moreno-Bayardo, M. G.,Torres, J., Jiménez, J. M. (2014). El discurso de los lectores académicos como mediación para formar investigadores. Jalisco, México: Universidad de Guadalajara.

Moreno, M. G. (2002). Formación para la investigación centrada en el desarrollo de habilidades. Guadalajara: Universidad de Guadalajara. 
Mullins, G. y Killey, M. (2002). It's a PhD, not a Nobel Prize': how experienced examiners assess research theses ["Es un doctorado, no un premio Nobel": cómo examinadores experimentados evalúan tesis de investigación]. Studies In Higher Education, 4(7), 369-386. doi: https://doi.org/10.1080/0307507022000011507

Piñuel, J. L. (2002). Epistemología, metodología y técnicas del análisis de contenido. Estudios de Sociolingüística, 1(3),1-42. España: Universidad Complutense de Madrid.

Ratcliffe, J. W. y González-Del Valle, A. (2000). El rigor en la investigación de la salud: Hacia un desarrollo conceptual. En C. Denman y J. Haro (Eds.), Por los rincones. Antología de métodos cualitativos en la investigación social (pp.57-112). Hermosillo, Sonora, México: El Colegio de Sonora.

Rodenes, M., Chismol, R. y Arango, M. D. (2000). Un enfoque sistemático para realizar la tesis doctoral. Psicothema, 2(12), 474-478.

Sautu, R. (2001). Acerca de qué es y no es investigación científica en ciencias sociales. En C. Wainerman y R. Sautu (Comps.), La trastienda de la investigación (pp. 227-243). Argentina: Lumière.

Stake, R. (2006). Multiple Case Study Analysis [Análisis de estudio de caso múltiple]. New York: The Guilford Press.

Stoilescu, D. y Mc Dougall, D. (2010). Starting to publish academic research as a doctoral student [Comenzando a publicar investigación académica como estudiante de doctorado]. International Journal of Doctoral Studies, 5 , 79-91. doi: https://doi.org/10.28945/1333

Taylor, S. J. y Bogdan, R. (1992). Introducción a los métodos cualitativos de investigación. Barcelona: Paidós.

Torres, J. (2013). Relación de tutoría y promoción del desarrollo de habitus científicos en estudiantes de doctorado en educación: Acercamiento a un caso. Perfiles Educativos, 35(140), pp. 8-27. doi: https://doi.org/10.1016/ S0185-2698(13)71819-1

Trafford, V. y Leshem, S. (2009). Doctorateness as a threshold concept [Doctoradad como un concepto innovador]. Innovations In Education and Teaching International, 3(3), 305-316. doi: https:// doi.org/10.1080/14703290903069027

Wainerman, C. (2001). Acerca de la formación de investigadores en ciencias sociales. En C. Wainerman, y R. Sautu (Comps.), La trastienda de la investigación (pp. 15-43). Argentina: Lumière.

\section{Notas}

[4] El título reportado de esta investigación es "El discurso de los lectores académicos como mediación para formar investigadores en el posgrado".

[5] The reported title of this research is "The Discourse of Academic Readers as Mediation in the Training of Postgraduate Researchers".

\section{BY-NC-ND}

